\title{
Bordes blandos
}

Jan Gehl

Recibido 2017.12.07 ::: Aceptado 2017.12.11

DOI: 10.5821/palimpsesto.17.5376

INTRODUCCIÓN (ABSTRACT) Y TRADUCCIÓN Alberto Peñín

El conocido arquitecto danés Jan Gehl (Copenhague 1936), lleva aquí su trayectoria de investigación sobre los espacios públicos a analizar el valor que tienen los que denomina "bordes blandos" ("soft edges") de las que denomina "bordes blandos" ("soft edges") de las áreas residenciales. Bordes blandos que incluyen los
patios frontales, porches, patios semiprivados, etc. que adquieren, tras una serie de estudios y encuestas realizados en paralelo en calles residenciales de Australia, Canadá y Escandinavia, un papel importante en la capacidad de dotar de vida, de animación, a los espacios públicos de estas áreas.

Este sugerente artículo nos remite al interés por lo cotidiano más que por los contenidos ideológicourbanísticos, como herramienta de conocimiento de la ciudad y de sus usuarios -y por tanto como instrumento de diseño -, que Jan Gehl nos ha acostumbrado desde sus primeros trabajos ("Life between buildings", 1971) y que en esta entrega en el Scandinavian Housing and Planning Research (1086) nos ampliaba hacia la tipificación de los espacios de borde, sus usos tipificación de los espacios de borde, sus usos posibles sus potenciales grupos sociales, vinculandola a la forma y características de esos bordes blandos entre la viviend y la propia calle. La carga propositiva que contiene esta investigación se refleja en otros trabajos, a partir de sus célebres tres condiciones para hacer más habitable la ciudad y de mejor calidad su vida urbana: andar más, estar más tiempo en la calle y salir de casa (que hizo suyas Copenhague); acompañada de una amplísima na amplisima labor de divulgación; "La humanización del espacio público", "Ciudades para la gente"," Nuevos espacios públicos", "Cómo estudiar la vida pública", que sitúa siempre la valoración de la ciudad y de su espacio público en el papel que en ella juega el ser humano.

El diagnóstico que Gehl realizaba en este texto en lo referente tanto a la estructura social como en los hábitos de comportamiento tiene hoy una renovada vigencia. Asimismo, la atención por los espacios frontera nos remite a la idea contemporánea de dilución de los límites que en climas como el nuestro incorpora la noción del espacio climas como el nuestro incorpora la noción

Todo ello, la escasa divulgación entre lectores de habla hispana y la no aparición de estudios similares en países más proclives al contacto social y utilización de espacios públicos como los latinos, nos anima a reproducir su contenido con la esperanza de despertar el interés de nuestros estudiantes e investigadores.

\section{La calle residencial}

La calle residencial es el objeto de estudio y discusión de este artículo. La razón para interesarse por este tipo de calle es el hecho de poder encontrarse en cas cualquier área residencial. Proporciona acceso, es un espacio potencialmente público y compartible.

Como el número de personas por hogar está a la baja en las sociedades industrializadas, como las áreas residenciales tienden a integrar cada vez menos usos (tiendas, servicios, y talleres), el número de potenciales usuarios de los espacios exteriores está asimismo decreciendo. Dicho de otra manera, ya no hay tanta gente y tampoco hay tantas actividades generadas. Así el número de espacios públicos abiertos se ha de limitar para garantizar, al menos, una cantidad razonable de vida y oportunidades sociales para crear un espacio compartido, es decir público. Existe por tanto la necesidad de poner el foco en las calles como tales y como lugares para albergar vida social.

Las calles residenciales estudiadas se caracterizan por (1) tráfico ligero, (2) edificios de pocas (1-2-(3-4)) alturas, (3) tramas edificadas algo densas de ciudad o casas aterrazadas (o apartamentos de baja altura), y (4) por puertas de entrada frontales a las calles. Las calles discutidas son pues calles que, debido a la razonable densidad de vivienda de baja altura (permitiendo un fácil acceso a las áreas exteriores y así maximizando las visitas exteriores por hogar), y ligero tráfico local, están muy probablemente destinadas a funcionar como un espacio público compartido y vivo para los residentes.

\section{La vida en las calles residenciales}

Una multitud de estudios apuntan al hecho de que la vida y las actividades en las calles y en varios tipos de espacios públicos están ampliamente considerados como un atractivo y tienen una apreciada calidad.

En áreas urbanas, las calles y plazas con vida se prefieren generalmente a aquellas que no la tienen. Los bancos con las mejoras vistas a la animación y actividades son los más utilizados. Encontraremos a niños allá donde una y otra estén a mano. Así, "la gente tiene tendencia a ir donde hay gente". [...]

En relación con las calles residenciales, los estudios centrados en patrones de juego de los niños, el uso de los bancos, el uso de los porches y de los patios de acceso, han enfatizado las citadas conclusiones por las cuales la vida y las actividades constituyen una importante y muy apreciada calidad en estas áreas.

Con los resultados de este mismo estudio y de otros similares, la existencia de una animada calle residencial, un espacio frecuentemente utilizado por otras personas, un lugar donde la gente y diversas actividades están a mano para la participación, para la inspiración o simplemente para contactos pasivos -mirar y escuchar- es muy valorada. La otra cara de la moneda- espacios sin vida o seguridad, espacios aislados, aburridos o sin compañeros de juego, etc- no tiene una valoración positiva.

En este contexto, se presenta una completa relación de argumentos, teorías e investigaciones que enfatiza las virtudes de una cierta cantidad de vida en las calles residenciales. Tiene un razonable sentido dejar a los miles y miles de amplios y pequeños eventos en la calle que hablen por sí solos.

Un buen número de estudios muestran cómo los sentimientos de territorialidad y pertenencia pueden estar relacionados directamente al grado de uso y vida en calles residenciales, algo estrechamente vinculado al sentimiento de seguridad, y a la actual protección respecto a la violencia y el vandalismo (Comité de Prevención del Crimen, Dinamarca, 1984).

\section{Animación en las calles, ¿una cuestión} de importancia creciente?

Naturalmente el énfasis en la presencia o ausencia de vida en las calles residenciales puede ser vista como una cuestión de ideología, o estilo de vida y preferencias. [...] Así, el acceso a calles animadas y al encuentro con otros en un área residencial podría hoy carecer de importancia.

Otros argumentos, sin embargo, conducirían a una conclusión diferente. Un número de cambios habidos en sociedades industriales occidentales apuntan hacia la creciente importancia del acceso a espacios animados en las áreas residenciales contemporáneas. Señalaremos:

(a) La reducción de la dimensión de los hogares. La media de los hogares en Dinamarca ha caído de 4,7 en 1900 a 2,1 personas en 1985. En las ciudades, el tamaño medio de los hogares será incluso menor (Copenhague $1,75)$. Más de la mitad de los hogares daneses son de una o dos personas. Así una serie de funciones sociales que antes se representaban en el interior de los hogares ahora discurren fuera de ellos.

(b) Los cambios en la estructura de edad de la población. Hay generalmente menos niños proporcionalmente a la población y menos niños en cada familia. Los compañeros de juego -y las áreas de juego/ espacios públicosdeberían ser hoy más accesibles - y las casas estar más cerca entre sí. Otro cambio es el creciente número de gente mayor. Este grupo está caracterizado por tener una considerable cantidad de tiempo libre y muchos años de buena salud y vida activa centrada alrededor de su vivienda. No se ha encontrado grupo alguno que haya usado más los espacios públicos y las calles animadas que este creciente grupo de gente mayor.

(c) Cambios en el carácter de las situaciones laborables. Un gran número de personas tendrá un trabajo más monótono, menos satisfactorio y menos creativo debido a la automatización y al desarrollo tecnológico. Esto tiende a invertir la primacía del espacio de trabajo frente al de la vivienda para una serie de actividades sociales y creativas.

(d) Finalmente, la tecnología y otros avances tienden a reducir el número total de horas de trabajo necesarias para una sociedad. Así el tiempo y la energía se liberan del trabajo y en consecuencia se desplaza al ámbito de la vivienda.

En suma, estos cambios implicarán que más personas (viviendo en hogares todavía más pequeños) tengan más energía, recursos y tiempo disponible y que sus necesidades sociales y creativas necesiten nuevas salidas. [...]

Estudios sobre una serie de calles y plazas del centro de Copenhague llevados a cabo en 1968 y de nuevo en 1983 muestran claramente que el uso de los espacios públicos ha aumentado marcadamente durante estos 15 años (mientras que la población de Copenhague
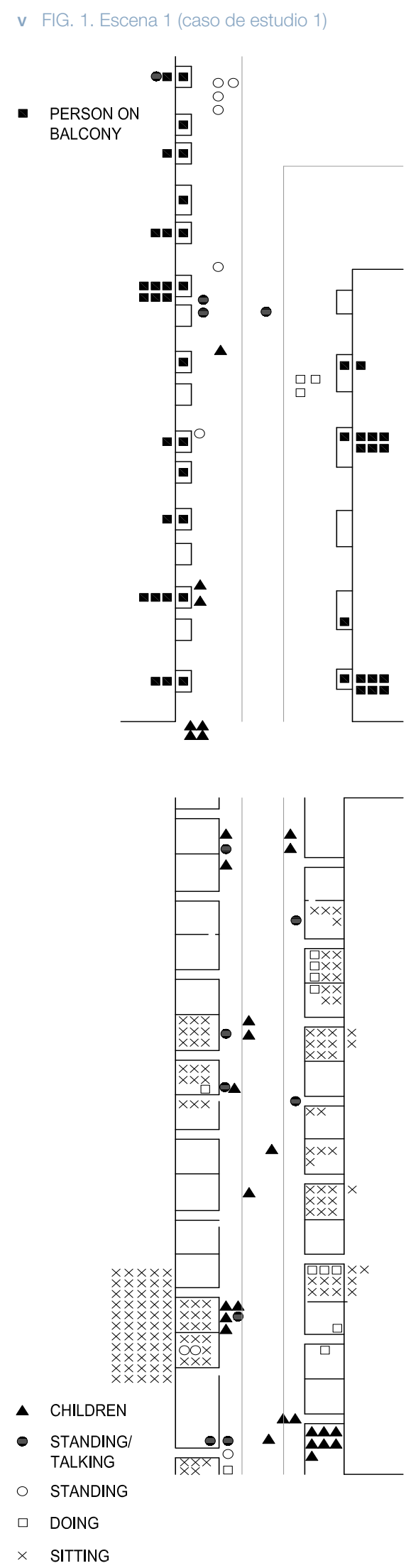

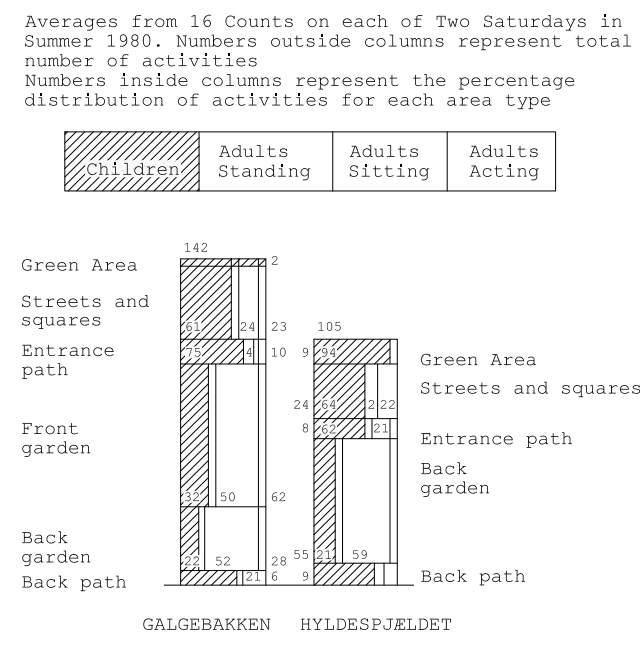

FIG. 2. Escena 2 (caso de estudio 2)

ha disminuido). Son utilizados por más personas y existe más variedad en sus actividades. Más aún, éstas han tomado una clara dirección hacia una mayor creatividad y expresividad como la música, el teatro, los grupos políticos e ideológicos, malabaristas, animadores y otros actores. [...

En este contexto, procede el estudio de algunos de los problemas relativos a la creación de calles residenciales animadas.

\section{Estancias de larga duración hacen} calles animadas

La duración de las actividades en la calle residencial es de lejos el factor más importante en relación al éxito o a fracaso de la animación en una calle.

En este contexto, "vida" se define como "personas presentes en la calle" - una definición cercana a lo que uno de hecho experimenta: una calle animada es una calle con presencia de gente- una calle sin vida es una calle sin gente. Una persona que pase 30 minutos en la calle equivaldrá a 30 personas pasando cada una de ellas un minuto. Así, no importa tanto el número total de personas, sino el número total de minutos pasados en las calles. [...]

La calle principal está llena de vida porque muchas personas acuden allí, cada una ellas un tiempo corto. La plaza central con muchos cafés en las aceras es particularmente viva porque los clientes de los cafés pasan mucho tiempo all.

La calle residencial está generalmente caracterizada por el hecho de que solo un número limitado de personas, potenciales usuarios, vive en cada calle. Así con una relativa baja densidad de viviendas y personas en las áreas residenciales, la única manera en la que la vida en las calles puede ser sustancialmente mejorada será incrementando la duración de las estancias.

Los niños pasan tiempo jugando en la calle, si la calle permite el juego. Los adultos pasan tiempo en la calle, si tienen algo que hacer y hay lugares para sentarse. El tráfico, es decir las personas yendo o conduciendo hacia o desde sus viviendas, no tiene mucha repercusión en este contexto. [...]

En 1977 un estudio de 12 calles residenciales con casas aterrazadas o urbanas en Waterloo (Ontario) y alrededores, enfatizaba la importancia del "tiempo pasado" en las calles como el factor clave para medir la animación. La observación directa de las actividades diurnas en las 12 calles concluye que el $52 \%$ de todas las actividades desarrolladas estaban relacionadas con el tráfico -entrando y saliendo-, mientras que el resto se refería a gente hablando, estando o haciendo alguna cosa, o a niños jugando.

Más tarde se concluyó que las actividades de tráfico generalmente son de duración muy breve. Aparcar y entrar en una vivienda duraba entre 20 y 30 segundos. Caminar a una casa solo duraba entre uno y dos minutos, medido como tiempo pasado en la calle. Por otra parte, hablar, estar, hacer alguna cosa, o jugar solía durar mucho más. La duración media de las actividades individuales era: hablar 3 minutos, estar 11 minutos, hacer 11 minutos, jugar 13 minutos. Combinando e número de personas con el tiempo pasado en varias actividades se concluyó que el 89 \% de toda "la vida en las calles" implicaba hablar, estar, hacer y jugar, mientras que entrar y salir registraba tan solo un mero 11 por ciento de esa "vida". [...]
Los elementos físicos que pueden soportar directamente estas actividades de "larga duración" -y que por tanto afectan directamente a "la vida en las calles"- han sido investigados en un estudio de 17 calles en y alrededor de Melbourne. Los hallazgos más relevantes de este estudio -basado en diversas técnicas de obsenvaciónfueron que el $70 \%$ de las actividades de "larga duración" encontradas se desarrollaban en los espacios delanteros semiprivados , mientras que solo el 30\% lo hacían en la parte exterior o en cualquier otra parte de la calle. Otro hallazgo importante tenía que ver con la disposición y detalle de estos patios frontales. [...] Los patios frontales que eran demasiado estrechos o con escasas espacios para sentarse eran considerablemente menos usados. Asi, para ser útiles, para albergar vida en las calles los espacio delanteros han de ser susceptibles de albergar actividade de larga duración, una cualidad muy bien desarrollada en la mayoría de los antepatios australianos que bordean las calles de las casas tradicionales aterrazadas.

Considerado en su conjunto, este estudio apuntaba contundentemente a los patios frontales semiprivados -los "bordes blandos"- como un elemento físico de gran importancia para albergar la vida en las calles.

En 1980-81 una serie de estudios comparativos que concernieron el gran Copenhague profundizaron en esto aspectos. En este texto presentamos dos de los casos estudiados.

\section{Dos casos de estudio}

Escena 1. Dos calles paralelas en un suburbio interior de Copenhague

Las dos calles se construyeron alrededor de 1940. Las dos tienen el mismo tipo de pisos y rentas y grupos de habitantes comparables. La calle A tiene edificios con 4 pisos y pequeñ pisos y pequeños balcones mientras que la calle $B$ tiene edificios con 3 pisos. La mayor diferencia entre ambas es que la calle B tiene jardines frontales pertenecientes a los pisos de planta baja, creando un borde utilizable y "blando" en el espacio público, mientras que la calle A presenta un borde "duro" y ninguna oportunidad para permanecer en la calle. Ambas áreas tienen patios interiores, los situados en la calle A de terrible calidad.

La vida en la calle fue estudiada simultáneamente durante un determinado número de días en verano de 1980. Las técnicas empleadas fueron la observación y registro de todas las actividades en calles, patios y balcones, desde el alba hasta el crepúsculo. La calle A, con un pobre entorno físico sin prácticamente ninguna oportunidad para estar al exterior ni para niños ni adultos, resultó estar casi desértica. [...] La conclusión es que cuando la disposición es demasiado pobre, la mayoría de las personas sencillamente no abandonan sus casas, mientras que una minoría, un grupo más dinámico sale fuera a otras áreas, playas y parques.

Con patios frontales, la calle $B$ resultó ser mucho más viva durante los mismos días. Dicho todo ello, la actividad (número total de minutos pasados) en la calle $B$ resultó ser 21 veces mayor que la generada por el mismo número de hogares en la calle A. Los hallazgos más detallados mostraron que los patios frontales fueron ampliamente usados por los adultos. [...] Los niños se encontraban raramente en los espacios delanteros comunes. Casi todos ellos estaban en las aceras y en la propia calle. Más aún, la intensa actividad dentro y alrededor de los jardines frontales provocaba que bastante gente de los dos pisos superiores fuera al escenario de la calle. Así pues, "donde la gente está, la gente acude". El alto nivel de actividad en esta calle no fue solo causado por más gente en el espacio público, sino especialmente por el hecho de que pasaban mucho más tiempo allí gracias a la mayor provisión de dispositivos para estar y sentarse. [...]

El caso de la calle A muestra que, si la calidad es demasiado baja, nadie utilizará los espacios públicos salvo por necesidad. Entrar y salir son prácticamente los únicos eventos que suceden en ellos. El caso de la calle B subraya la enorme diferencia que los adecuados dispositivos para la estancia pueden suponer para una calle. Los habitantes de todas las generaciones tienen a su disposición un lugar al que ir y algún sito para estar. Más aún, pueden sacar a la calle sus diversas actividades domésticas. [...]

Escena 2. Dos áreas de vivienda en un suburbio al sur de Copenhague

"Hyldespjaeldet” y "Galgebakken" son dos áreas residenciales de "densidad media y baja altura". Ambas fueron construidas entre 1972 y 1975 , son de vivienda pública de alquiler, y fueron ocupadas en el mismo período de tiempo y por grupos de habitantes similares. Más aún, las dos áreas son de tamaño similar y próximas una a otra.

La asombrosa diferencia entre las dos tiene que ver con la existencia solo en "Galgebakken de un patio frontal semiprivado, cuidadosamente diseñado y detallado, a modo de zona intermedia entre las viviendas y los carriles de acceso público" [...].

Con la mayoría del resto de factores lo más estables posible los espacios exteriores disponibles son:

\section{Hyldespjaeldet Galgebakken}

Parque/ verde comunitario Parque/ verde comunitario Calles y plazas

Carriles de acceso

Jardines privados traseros Patios frontales/ espacios

Carriles traseros

\section{delanteros}

Jardines privados traseros

Carriles traseros

Uno tiene espacios delanteros semiprivados, el otro no. ¿Habría una diferencia en los patrones de actividad en estas dos áreas?

Este fue el objeto de otra serie de estudios realizados simultáneamente durante el verano de 1980. [...] Las principales conclusiones fueron que, en el área con los patios frontales, un 35\% más de personas utilizaron espacios exteriores que en la otra área con el mismo número de hogares, pero sin patios frontales. [...] La existencia en un área de una más variada y mejor diseñada matriz de espacios exteriores - y especialmente la provisión de un muy utilizable y semiprivado patio de acceso- condujo a un considerable mayor nivel de actividades en las áreas exteriores. [...]

Un sorprendente hallazgo sobre las áreas frontales fue que eran utilizadas dos veces más que los patios traseros. Dada la opción entre quedarse en un patio trasero recluido o quedarse en un patio frontal abierto y semiprivado en el lado público de las casas, esta última fue elegida con el doble de frecuencia que la primera. [...]

Es realmente interesante constatar que cuando se proporcionan estas oportunidades resultan ser intensamente utilizadas. [...] La existencia de espacios de transición semiprivados es muy utilizada por los habitantes en su paso entre sus casas privadas y el reino de lo público. Los patios frontales ayudaron a los habitantes a "tomar el primer y más difíil paso" desde sus casas, hasta los espacios compartidos.

\section{La vida en las calles residenciales}

...] Los resultados de los estudios en los tres continentes son similares. No debería ser una sorpresa, dada la semejanza de los dispositivos físicos y de los detalles. En cada caso, los residentes tuvieron acceso a un espacio bien dimensionado, un área, -por ejemplo, un porche o un patio frontal- en el lado de la calle de las casas. Que este dispositivo se emplee de diversas maneras no puede ser sorprendente. [...]

Más interesante, sin embargo, es la conclusión por la cual la disposición de un espacio extra en el límite entre la casa y la terraza generalmente parece ser un factor de refuerzo de la vida en las calles residenciales. Es todavía más interesante constatar que esta oportunidad es principalmente utilizada por los adultos, es decir un grupo que tradicionalmente tiene muy pocas oportunidades para quedarse en la cara pública de las casas por tiempo prolongado.

Los "bordes blandos" en sus diversas formas son asi definitivamente un factor de importancia significativa para determinar la existencia o no de "vida" en las calles residenciales, aunque sea uno de entre numerosos factores. Otros serían la densidad de las viviendas y habitantes, el tipo y la altura de los edificios, las dimensiones de las calles, los modos e intensidades del tráfico, las condiciones climáticas, el mobiliario urbano, así como factores relacionados con la cultura, el estilo de vida y las preferencias.

Destaquemos pues que, los "bordes blandos" como espacios muy importantes en la animación de las áreas residenciales, son un punto de partida evidente para acoger la vida en las mismas.

JAN GEHL es arquitecto (Royal Danish Academy of Fine Arts) y consultor de Diseño Urbano en Copenhagen (Gehl Architects). 